\title{
Nanoscale band gap spectroscopy on ZnO and GaN-based compounds with a monochromated electron microscope
}

\author{
M. Bosman, ${ }^{1, a)}$ L. J. Tang, ${ }^{1}$ J. D. Ye, ${ }^{1}$ S. T. Tan, ${ }^{1}$ Y. Zhang, ${ }^{1}$ and V. J. Keast ${ }^{2}$ \\ ${ }^{1}$ Institute of Microelectronics, A*STAR (Agency for Science, Technology and Research), \\ 11 Science Park Road, Singapore 117685, Singapore \\ ${ }^{2}$ School of Mathematical and Physical Sciences, The University of Newcastle, Callaghan, \\ New South Wales 2308, Australia
}

(Received 1 August 2009; accepted 16 August 2009; published online 11 September 2009)

\begin{abstract}
Monochromated low-loss EELS (electron energy-loss spectroscopy) is explored as an analytical technique for nanoscale mapping of the electronic band gap energy on arsenic-implanted $\mathrm{ZnO}$, $\mathrm{CdZnO}$, and InGaN compounds. Its accuracy is confirmed independently with Raman spectroscopy. From a ternary compound, the relationship between the band gap energy and the chemical composition is determined, a powerful application of low-loss EELS. The effects of electron beam delocalization are discussed using examples from $\operatorname{In}_{0.25} \mathrm{Ga}_{0.75} \mathrm{~N}$ quantum wells. () 2009 American Institute of Physics. [doi:10.1063/1.3222974]
\end{abstract}

Chemical analysis of materials with nanometer spatial resolution can be done with a (scanning) transmission electron microscope $[(\mathrm{S}) \mathrm{TEM}]$ using the spectroscopic techniques of EELS and EDX (electron energy-loss spectroscopy and energy-dispersive $\mathrm{x}$-ray spectroscopy). With the recent commercial availability of electron monochromators in (S)TEMs, it has also become possible to probe electron transitions in the visible and near-UV spectral range. ${ }^{1}$ It is only a matter of time before these new instruments will be widely used for the study of optically active materials. In this letter, we will explore the possibilities and limitations of monochromated low-loss EELS for measuring the electronic band gap energy in the nanometer spatial domain. Examples will be given from wurtzite $\mathrm{ZnO}$ - and $\mathrm{GaN}$-based nanostructures, as these direct band gap semiconductors are of significant technological interest.

The experiments in Figs. 1 and 2 on $\mathrm{ZnO}$ were done with a monochromated FEI Titan, operated at $80 \mathrm{kV}$-near the Čerenkov limit of $\mathrm{ZnO}$ - to avoid strong, unwanted retardation losses in the EELS spectrum. ${ }^{2}$ The STEM probe had a diameter around $1 \mathrm{~nm}$, and the EELS collection angle of 25 mrad gave an energy resolution better than $0.12 \mathrm{eV}$ (measured by the full width at half maximum of the zero-loss peak). TEM specimens for band gap measurements were prepared with focused ion beam (FIB), cleaned to a thickness of $\sim 50 \mathrm{~nm}$ with $2.5 \mathrm{kV}$ helium ions. Additional tripodpolished and argon-ion milled specimens were prepared (not shown) that quantitatively reproduced the measurements on the FIB-prepared specimens. This indicates that the specimen preparation methods did not modify the inherent band gap values.

It is known that the electronic properties of crystalline $\mathrm{ZnO}$ can be modified by the introduction of point defects. ${ }^{3,4}$ For this work, defects were introduced by implanting arsenic ions into hydrothermally grown, ${ }^{5}$ single crystal $\mathrm{ZnO}$. At room temperature, $150 \mathrm{keV} \mathrm{As}^{+}$ions were homogeneously implanted with a fluence of $5 \times 10^{15} \mathrm{~cm}^{-2}$, tilting the samples $7^{\circ}$ relative to the incident beam to minimize chan-

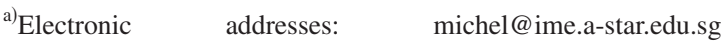

and neling effects. Under these conditions, arsenic was implanted in the first $150 \mathrm{~nm}$ below the surface of the $\mathrm{ZnO}$, as shown in Fig. 1. The inset shows resonant Raman spectra, obtained using $325 \mathrm{~nm} \mathrm{He}-\mathrm{Cd}$ laser excitation at room temperature. After arsenic implantation, the broad peak of hot luminescence has blueshifted by $0.14 \mathrm{eV}$.

The EELS band gap profile of the arsenic-doped $\mathrm{ZnO}$ (blue squares) confirms the Raman measurement by showing a band gap increase of around $0.14 \mathrm{eV}$ in the first $\sim 150 \mathrm{~nm}$. Thermal annealing gradually decreased the band gap energy to bulk $\mathrm{ZnO}$ values. The exact origin of the blueshift remains unknown, but it has been suggested that its origin lies in quantum confinement effects due to $\mathrm{ZnO}$ nanocrystallinity introduced by the arsenic implantation. ${ }^{6}$ The confirmation of the EELS band gap measurement by an independent technique (resonant Raman spectroscopy) is reassuring. A next step is to combine band gap and EDX measurements.

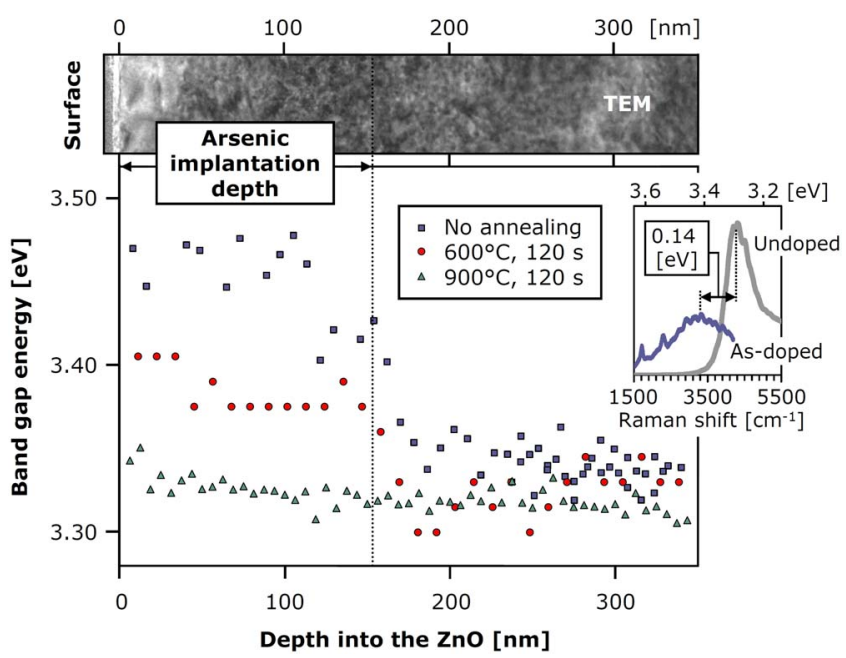

FIG. 1. (Color online) Top: TEM bright field image of arsenic-implanted $\mathrm{ZnO}$ before annealing. Bottom: The electron band gap profiles were measured with monochromated EELS, on an arsenic-implanted specimen without subsequent annealing (blue squares), on a specimen with 2 min annealing at $600{ }^{\circ} \mathrm{C}$ (red circles), and on a specimen with 2 min annealing at $900{ }^{\circ} \mathrm{C}$ (green triangles) in ambient air. The inset shows resonant Raman spectra from undoped $\mathrm{ZnO}$ and arsenic-implanted $\mathrm{ZnO}$ (without annealing). 


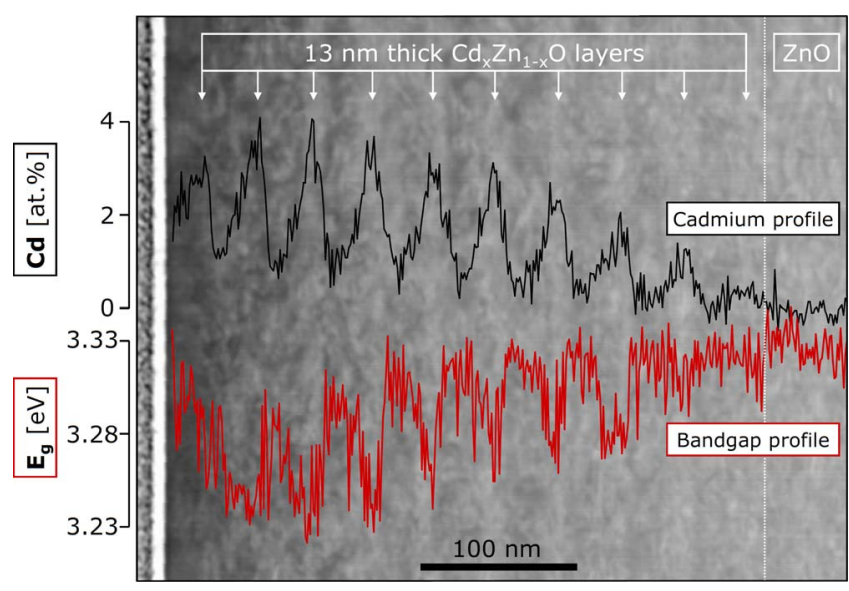

FIG. 2. (Color online) STEM annular dark field (ADF) image of the $\mathrm{Cd}_{x} \mathrm{Zn}_{1-x} \mathrm{O} / \mathrm{ZnO}$ multilayer with projected cadmium (black) and band gap (red) profiles. The $\mathrm{ZnO}$ substrate is indicated at the right, the specimen surface is on the left-hand side, marked by the bright platinum band from FIB specimen preparation.

It is known that by introducing cadmium as a ternary compound-forming element, the $\mathrm{ZnO}$ band gap energy can be tuned between 3.3 and $3.0 \mathrm{eV}^{7}$ The relation between the cadmium fraction $x$ and the band gap energy is studied here on a $\mathrm{Cd}_{x} \mathrm{Zn}_{1-x} \mathrm{O} / \mathrm{ZnO}$ multilayer with increasing $x$. A Thomas Swan closed-coupled showerhead metalorganic chemical vapour deposition (MOCVD) system was used for specimen growth at $850{ }^{\circ} \mathrm{C}$, using dimethylzinc and dimethylcadmium as the respective zinc and cadmium sources. To ensure epitaxial quality, the layers were deposited on undoped $\mathrm{ZnO}$ templates with excellent crystal quality and smooth surface morphology. Ten $\mathrm{Cd}_{x} \mathrm{Zn}_{1-x} \mathrm{O}$ layers of 13 $\mathrm{nm}$ thickness and increasing cadmium content were thus formed, sandwiched between $25 \mathrm{~nm}$ thick, undoped $\mathrm{ZnO}$ layers, as shown in Fig. 2.

Following the band gap measurements, the microscope was realigned at $300 \mathrm{kV}$ and from the same area, an EDX profile was taken with a STEM probe of less than $0.3 \mathrm{~nm}$ in diameter. Quantification of the cadmium content was done with standardless, thickness-corrected EDX using calculated $k$-factors. This gave for the spectra with highest Cd signal an atomic percentage of $4.0 \pm 1.2 \%$. Even though the error in quantification is relatively large due to the uncertainty in ionization cross section for cadmium, it agrees with earlier optical absorption measurements ${ }^{7}$ that found maximum cadmium miscibility in $\mathrm{ZnO}$ at 4-7 at. \%. For the specimen in Fig. 2, it can be seen that the morphology of the material becomes less homogeneous with higher cadmium content, indicating that the last two $\mathrm{Cd}_{x} \mathrm{Zn}_{1-x} \mathrm{O}$ layers have decomposed. Another observation is that the cadmium content does not reach zero in the center of the $\mathrm{ZnO}$ layers, particularly for the ones closest to the surface side. This indicates some cadmium intermixing in the $\mathrm{ZnO}$ layers; the lower band gap profile also supports this conclusion.

A specific advantage of spatially resolved band gap profiling over optical spectroscopy now becomes clear: from the same sample area, the cadmium fraction is known, as well as the band gap energy and the layer quality (from the STEM image). It is now possible to quantitatively correlate the cadmium content with the band gap energy. An advantage over optical absorption spectroscopy is the availability of direct information on structural homogeneity; layers that have de-
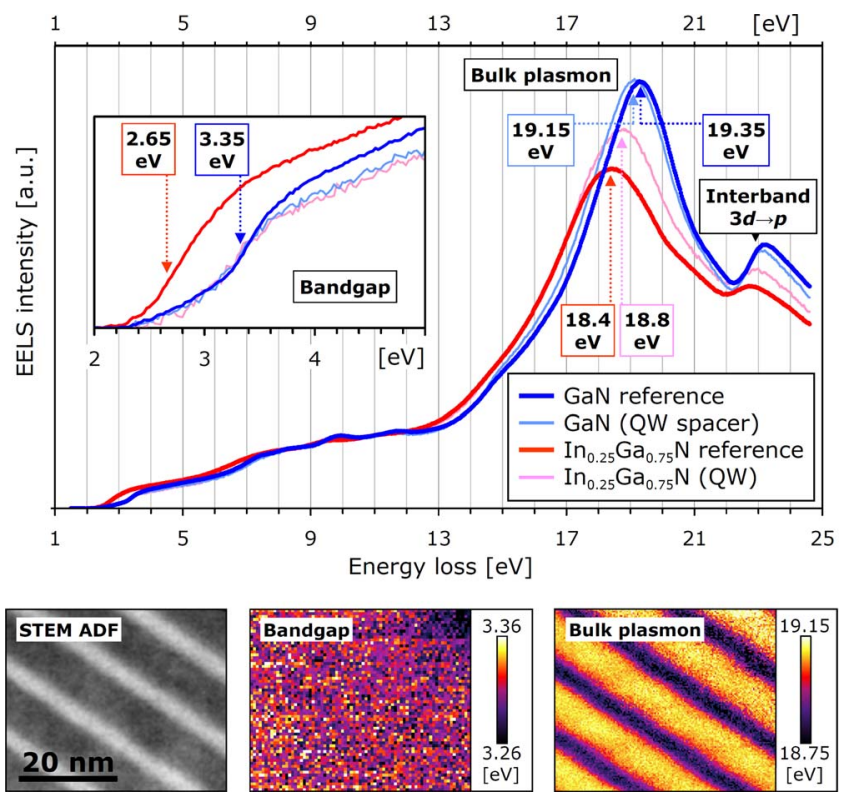

FIG. 3. (Color online) Low-loss EELS spectra taken from the central $1.5 \mathrm{~nm}$ of an $\mathrm{In}_{0.25} \mathrm{Ga}_{0.75} \mathrm{~N}$ quantum well (pink) and from the $\mathrm{GaN}$ spacers between the quantum wells (light blue), compared with bulk $\operatorname{In}_{0.25} \mathrm{Ga}_{0.75} \mathrm{~N}$ (red) and $\mathrm{GaN}$ reference spectra (dark blue). The spectrum inset shows a closeup of the band gap features. The lower part of the figure shows a STEM ADF image, taken with the same probe settings as the adjacent EELS maps, which image the band gap energy (binned twice) and the bulk plasmon energy of the four quantum wells.

composed into phases of high and low cadmium fractions can be removed from the data set. If the two ${ } d_{x} Z_{1-x} \mathrm{O}$ layers closest to the surface are rejected for analysis, the relation between the cadmium fraction $x$ and the band gap is determined to be $E_{g}=3.32-2.43 x-8.43 x^{2}$ ( $R^{2}$ value is 0.95$)$. However, this traditional second-order relation can be approximated with a comparable goodness-of-fit $\left(R^{2}\right.$ value is $0.94)$ by $E_{g}=3.31-1.94 x$. As this covers the whole range of cadmium miscibility in $\mathrm{ZnO}$, the latter equation should be a useful rule-of-thumb for predicting the room temperature band gap energy for known $\mathrm{Cd}_{x} \mathrm{Zn}_{1-x} \mathrm{O}$ compositions, or vice versa.

What are the fundamental limitations of EELS band gap measurements? The strongest restrictions are imposed on the spatial resolution and energy accuracy, which will be studied here with the help of bulk and quantum confined $\mathrm{In}_{0.25} \mathrm{Ga}_{0.75} \mathrm{~N}$. ${ }^{9,10}$ The measurements of Fig. 3 were done with a VG STEM-Gatan Enfina system operated at $100 \mathrm{kV}$, where the electron extraction voltage at the cold field emission gun was greatly reduced to enhance the monochromaticity of the electron beam to $0.25 \mathrm{eV}$. The STEM probe diameter was smaller than $1.5 \mathrm{~nm}$ and EELS was acquired in spectrum image mode ${ }^{11,12}$ with a collection angle of 6 mrad, using binned gain averaging. ${ }^{13}$ TEM specimens were prepared by tripod polishing followed by argon ion milling. Indium-rich quantum wells with thickness $3.2 \mathrm{~nm}$ are spaced by $6.8 \mathrm{~nm}$ thick GaN layers, a structure similar to that of commercially available light-emitting diodes. ${ }^{14}$ The composition of the quantum wells was determined to be $\operatorname{In}_{0.25} \mathrm{Ga}_{0.75} \mathrm{~N}$, by scanning over a $\sim 10 \times 10 \mathrm{~nm}$ area, measuring the EDX signal and correcting for the fraction of the area that was occupied by the indium-containing quantum wells. ${ }^{14}$ Bulk measurements were done on $\operatorname{In}_{0.25} \mathrm{Ga}_{0.75} \mathrm{~N}$ that was epitaxially grown as a thick layer onto GaN. 
Quantum confinement and strain-induced piezoelectric polarization in the $3.2 \mathrm{~nm}$ thick $\mathrm{In}_{0.25} \mathrm{Ga}_{0.75} \mathrm{~N}$ layers is expected to increase the band gap energy from 2.65 to about $3.1 \mathrm{eV} .{ }^{15}$ Indeed, the band gap energy of the quantum wells, shown as the pink spectrum in Fig. 3, seems much higher than that of bulk $\operatorname{In}_{0.25} \mathrm{Ga}_{075} \mathrm{~N}$. However, the measured value of $3.35 \mathrm{eV}$ is larger than expected and also overlaps suspiciously with the GaN spectra. Here, electron beam delocalization ${ }^{16,17}$ is the source for possible misinterpretation; the lower the energy loss, the larger the distance over which a fast electron will excite specimen electrons. This delocalization degrades the spatial resolution for band gap measurements. In addition, it might easily be overlooked that for embedded, low-dimensional structures such as quantum wells, delocalization also degrades the energy-accuracy of the spectral features. For low-energy excitations such as band gap measurements, a large part of the signal will come from the adjacent $\mathrm{GaN}$ due to delocalization. For higherenergy transitions, such as the excitation of the bulk plasmon around $19 \mathrm{eV}$, the effective probe diameter is already much smaller. Yet, in the quantum well structure of Fig. 3, even the plasmon peak is a combination of quantum well and $\mathrm{GaN}$ plasmon signals. Using the epilayer specimens, the effective probe diameter was estimated by monitoring the distance to the interface where the spectral features appear to shift in energy. For the plasmon peak, an effective probe diameter of $4 \mathrm{~nm}$ was found; this increased to $10 \mathrm{~nm}$ for the band gap energy. The actual delocalization distance will be somewhat smaller, as the effective probe diameter includes the broadening effect of the $1.5 \mathrm{~nm}$ experimental beam.

The EELS maps of the band gap and plasmon energies in Fig. 3 were extracted using principal component analysis. ${ }^{18}$ It can be seen that the band gap energy map is featureless, and that the plasmon map only covers a limited energy range due to delocalization. It might be tempting to use low-loss EELS to map intrinsic local physical properties, but electron beam delocalization always needs to be considered. The larger dimensions of the structures in the earlier $\mathrm{ZnO}$ examples did not introduce signal from adjacent layers. For example, in the central 3-5 nm of the $\mathrm{Cd}_{x} \mathrm{Zn}_{1-x} \mathrm{O}$ layers, no $\mathrm{ZnO}$ band gap signal was present. However, the quantum wells of Fig. 3 have such small dimensions that even in their center, there is a significant signal from the adjacent GaN. ${ }^{19,20}$ The examples in Fig. 3 demonstrate that for such small structures, low-loss EELS should not be used to quantitatively map band gap energies, dielectric properties, etc. as delocalization degrades the accuracy of the measured energies. Accurate band gap mapping in the (near-) visible will not be possible on embedded layers or particles with dimensions smaller than around $10 \mathrm{~nm}$. However, low-loss electron transitions might still be extracted with alternative specimen designs. Free-standing quantum dots, ${ }^{21}$ metal nanoparticles, ${ }^{22}$ or structures of interest that are embedded in very wide band gap materials will provide better spectral accuracy for low-loss EELS, without signal overlap from adjacent specimen material.

In summary, monochromated low-loss EELS was used for measuring the electronic band gap energy of direct semiconductors, with a spatial resolution of around $10 \mathrm{~nm}$. The technique proved useful for the determination of the relationship between chemical composition and band gap energy in ternary compounds. (S)TEM imaging can be done simultaneously with chemical analysis and band gap measurements, providing concurrent information on the specimen nanostructure, a great advantage over optical spectroscopy. Possible pitfalls of monochromated low-loss EELS were also briefly discussed.

M. J. Kappers (University of Cambridge, U.K.) is acknowledged for providing the InGaN specimens. The Electron Microscope Unit at the University of Sydney (Australia) is thanked for support during the VG STEM experiments.

${ }^{1}$ L. Gu, W. Sigle, C. T. Koch, J. Nelayah, V. Srot, and P. A. van Aken, Ultramicroscopy 109, 1164 (2009).

${ }^{2}$ M. Stöger-Pollach, Micron 39, 1092 (2008).

${ }^{3}$ F. Tuomisto, K. Saarinen, D. C. Look, and G. C. Farlow, Phys. Rev. B 72, 085206 (2005).

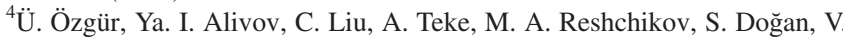
Avrutin, S.-J. Cho, and H. Morkoçd, J. Appl. Phys. 98, 041301 (2005).

${ }^{5}$ D. Schulz, S. Ganschowa, D. Klimma, and K. Struve, J. Cryst. Growth 310, 1832 (2008).

${ }^{6}$ J. D. Ye, S. Tripathy, F.-F. Ren, X. W. Sun, G. Q. Lo, and K. L. Teo, Appl. Phys. Lett. 94, 011913 (2009).

${ }^{7}$ T. Makino, Y. Segawa, M. Kawasaki, A. Ohtomo, R. Shiroki, K. Tamura, T. Yasuda, and H. Koinuma, Appl. Phys. Lett. 78, 1237 (2001).

${ }^{8}$ Th. Gruber, C. Kirchner, R. Kling, F. Reuss, A. Waag, F. Bertram, D. Forster, J. Christen, and M. Schreck, Appl. Phys. Lett. 83, 3290 (2003).

${ }^{9}$ V. J. Keast, A. J. Scott, M. J. Kappers, C. T. Foxon, and C. J. Humphreys, Phys. Rev. B 66, 125319 (2002).

${ }^{10}$ A. G. Gutiérrez-Sosa, U. Bangert, A. J. Harvey, C. J. Fall, R. Jones, P. R. Briddon, and M. I. Heggie, Phys. Rev. B 66, 035302 (2002).

${ }^{11}$ C. Jeanguillaume and C. Colliex, Ultramicroscopy 28, 252 (1989).

${ }^{12}$ J. A. Hunt and D. B. Williams, Ultramicroscopy 38, 47 (1991).

${ }^{13}$ M. Bosman and V. J. Keast, Ultramicroscopy 108, 837 (2008).

${ }^{14}$ M. Bosman, M. Sitarz, A. Z. Sikorski, and V. J. Keast, Appl. Phys. Lett. 84, 1371 (2004).

${ }^{15}$ P. Perlin, I. Gorczyca, T. Suski, P. Wisniewski, S. Lepkowski, N. E. Christensen, A. Svane, M. Hansen, S. P. DenBaars, B. Damilano, N. Grandjean, and J. Massies, Phys. Rev. B 64, 115319 (2001).

${ }^{16}$ D. A. Muller and J. Silcox, Ultramicroscopy 59, 195 (1995).

${ }^{17}$ R. F. Egerton, Ultramicroscopy 107, 575 (2007).

${ }^{18}$ M. Bosman, M. Watanabe, D. T. L. Alexander, and V. J. Keast, Ultramicroscopy 106, 1024 (2006)

${ }^{19}$ J. P. R. Bolton and M. Chen, Ultramicroscopy 60, 247 (1995).

${ }^{20}$ M. Couillard, M. Kociak, O. Stéphan, G. A. Botton, and C. Colliex, Phys Rev. B 76, 165131 (2007).

${ }^{21}$ R. Erni and N. D. Browning, Ultramicroscopy 107, 267 (2007).

${ }^{22}$ M. Bosman, V. J. Keast, M. Watanabe, A. I. Maaroof, and M. B. Cortie Nanotechnology 18, 165505 (2007). 